\title{
Induced Pluripotent Stem Cells: Global Research Trends
}

\author{
Takaharu Negoro, Hanayuki Okura, and Akifumi Matsuyama
}

\begin{abstract}
The induced pluripotent stem cell (iPSC) was first described more than 10 years ago and is currently used in various basic science and clinical research fields. The aim of this report is to examine the trends in research using iPSCs over the last 10 years. The 2006-2016 PubMed database was searched using the MeSH term "induced pluripotent stem cells." Only original research articles were selected, with a total of 3323 articles. These were classified according to research theme into reprogramming, differentiation protocols for specific cells and/or tissues, pathophysiological research on diseases, and discovery of new drugs, and then the trends over the years were analyzed. We also focused on 232 research publications on the pathophysiological causes of diseases and drug discovery with impact factor (IF; Thomson Reuters) of six or more. The IF of each article was summed up by year, by main target disease, and by country, and the total IF score was expressed as trends of research. The trends of research activities of reprogramming and differentiation on specific cells and/or tissues reached maxima in 2013/ 2014. On the other hand, research on pathophysiology and drug discovery increased continuously. The 232 articles with IF $\geq 6$ dealt with neurological, immunological/hematological, cardiovascular, and digestive tract diseases, in that order. The majority of articles were published from the United States, followed by Japan, Germany, and United Kingdom. In conclusion, iPSCs have become a general tool for pathophysiological research on disease and drug discovery.
\end{abstract}

Keywords: drug discovery; impact factor; induced pluripotent stem cells (iPSCs); pathophysiology of disease; PubMed; trends of research

\section{Introduction}

The first report that described induced pluripotent stem cells (iPSCs) was published more than 10 years ago. ${ }^{1}$ In the early days, iPSCs could be handled only by topranking researchers, but they are currently available to almost all researchers and used in various types of research fields. The aim of this research article is to investigate the phase transition of iPSC-based research.

One of the advantages of iPSCs compared with other kinds of stem cells is the availability to create cell models for diseases and based on that the pathophysiological research of diseases and drug discovery can be developed by reprograming cells from diseased patients and inducing cell differentiation suitable for evaluation. ${ }^{2}$ In this study, we searched and selected research articles that described work using iPSCs and were published between 2006 and recent years from the PubMed database and classified these publications according to their themes into reprogramming, differentiation protocols for specific cells and/or tissues, pathophysiological research of diseases, discovery of new drugs, and cell therapy, and then the trends over the years were analyzed. We also focused on the fields of pathophysiological research of diseases and drug discovery. In this

Platform of Therapeutics for Rare Disease, National Institutes of Biomedical Innovation, Health and Nutrition, Osaka, Japan.

*Address correspondence to: Hanayuki Okura, PhD, Platform of Therapeutics for Rare Disease, National Institutes of Biomedical Innovation, Health and Nutrition, Shogyo Bldg \#603, 5-6-10 Toyosaki, Kita-ku, Osaka 531-0072, Japan, E-mail: haookura-circ@umin.ac.jp

(c) Takaharu Negoro et al. 2017; Published by Mary Ann Liebert, Inc. This is an Open Access article distributed under the terms of the Creative Commons Attribution License, which permits unrestricted use, distribution, and reproduction in any medium, provided the original work is properly cited. 
regard, we used the impact factor (IF) score provided by Thomson Reuters. The IF score represents the average number of citations per article in a journal for 2 years since its publication and is used as an index to evaluate the journal. While IF itself does not directly reflect the scientific importance of the article, it has also been used, in general, as an index for evaluation of individual articles published in the journal, and the total IF score obtained by simply summing up a range of IF values has been used for relative comparison with others (e.g., Cao et al. ${ }^{3}$ ). It is thought that total IF score represents the expected score of the total number of citations in the range of those articles. Thus, in this study, we decided to use it as a rough index representing the quality $\times$ quantity of a given range of publication to evaluate trend of research on a particular main target disease or by nation.

\section{Methods}

To investigate the research trend of iPSCs, we searched the PubMed database for publications published between 2006 and 2016 ( August) using the MeSH term "induced pluripotent stem cells." However, since the term "induced pluripotent stem cells" was applied only after 2010, we also manually selected articles among those published between 2006 and 2009 and identified in the database with the search terms "induced pluripotent stem cells [All Fields]" OR "pluripotent stem cells [MeSH Major Topics]." To identify only original research articles, nonoriginal articles such as reviews, commentaries, news, and historical articles were excluded from the resultant article pool. Due to incomplete exclusion, some publications were deleted manually by further inspection of the articles. Articles that did not contain abstracts in the PubMed database were also excluded because it was impossible to evaluate such articles at a later stage. The 3323 indexed articles were later classified according to their themes into reprogramming, differentiation protocols for specific cells and/or tissues, pathophysiological research of diseases, discovery of new drugs, and cell therapy according to the following criteria:

(a) Reprogramming to iPSCs: all research articles related to reprogramming, such as genes, host cells, transfection methods, mRNA analysis, media, and feeder cells. Among these articles, we also selected

$\left(a^{\prime}\right)$ iPSC preparation protocols. Among (a), research articles that focused on iPSC preparation protocols were especially selected. (b) Differentiation protocols for specific cells and/or tissues and research: differentiation research using iPSCs, including protocols for specific cells and/or tissues and related research.

(c) Pathophysiological research of diseases: included research articles that used patient-derived and/ or disease-specific iPSCs in the title and/or abstract.

(d) Drug discovery: research articles that focused on applications to the so-called conventional drug discovery. Articles with only suggestion or without description of compound evaluation were excluded.

(e) Cell therapy: included research articles on application of iPSCs for cell therapy. Articles on antigenicity of iPSCs were also included in this category. In addition, when the article was considered to fall under multiple themes, we allowed up to two duplicates.

We obtained the IF from the Journal Citation Reports provided by Thomson Reuters, used the 2015 version data, indexed the IF score given to the individual journal to the corresponding article, and throughout this work, we handled them as IF scores of individual articles. In this work, the Journal Citation Reports covered about $93 \%$ of resultant articles selected from PubMed-listed journals. Journals that were not listed on the Journal Citation Reports were marked with $\mathrm{IF}=0$, about half of which were published in languages other than English.

The development stage was classified into three categories; basic research, applied research (drug screening or medicinal pharmacology), and translational research (preclinical and clinical tests). Articles were also classified according to countries of the corresponding author.

\section{Results}

To investigate the phase transition of iPSC-based research, we surveyed articles listed in the PubMed database. First, from the articles published between 2006 and 2016 ( August), we selected 3323 articles using the MeSH Term "induced pluripotent stem cells," excluding nonoriginal research articles, such as reviews. Figure 1A shows the number of articles classified according to their themes (reprogramming, differentiation, pathophysiological research of diseases, drug discovery, and cell therapy) and publication year (2006-2015). Figure 1B shows the total IF scores of the articles for each publication year 

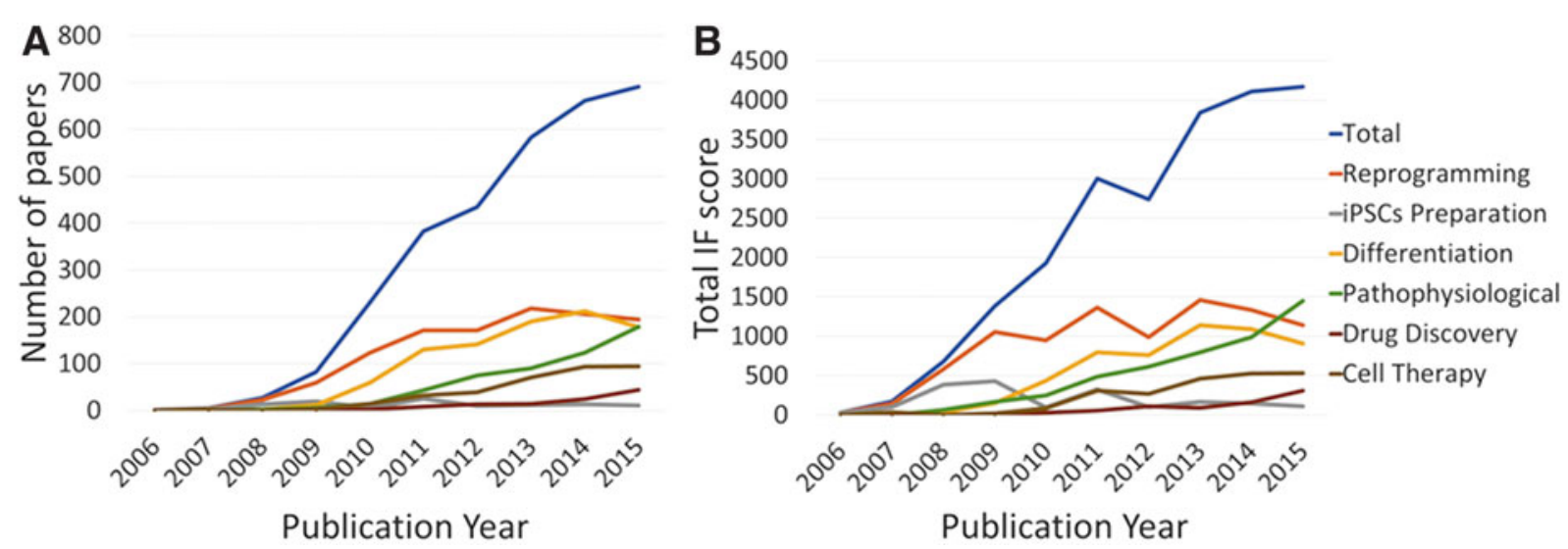

FIG. 1. (A) Number of iPSC-based research articles on PubMed database by publication year. (B) Total IF score of research articles on iPSCs stratified by publication year. The PubMed database was searched using "induced Pluripotent Stem Cells" as the MeSH term. In this graph, articles published in 2016 were not included since they could not include all articles published in the entire year. IF, impact factor; iPSC, induced pluripotent stem cell.

(2006-2015). The total IF score, which is the sum of IF of the articles of each year, represents the mass of quality $x$ quantity of each year.

The number of articles classified with the reprogramming theme reached a maximum at $n=218$ in 2013 and then decreased slightly in 2014 and 2015. Similarly, the total IF score reached a maximum at $s=1459$ in 2013 and then decreased to $s=1140$ in 2015. Analysis of articles with the theme of iPSC preparation protocol showed maximum number at $n=25$ in 2011, whereas the highest total IF score was shown at $s=433$ in 2009, and both did not exceed half of the peak value after 2012. For articles classified with differentiation theme, their number was less than that of reprogramming articles, reaching maximum at $n=212$ in 2014 and then decreasing in 2015. Their total IF score was highest at $s=1142$ in 2013 , then decreased in 2015 , similar to the reprogramming theme. On the other hand, the number of articles marked as dealing with pathophysiological research increased after lagging 3 to 4 years behind reprogramming articles, and continued to grow, and reached at $n=179$ in 2015, becoming almost equivalent to those with reprogramming and differentiation themes. As the total IF score rose to $s=1448$ in 2015, in contrast, the reprogramming and differentiation thesis were reduced to $s=1140$ and $s=1095$ respectively, so pathophysiological research thesis showed the highest total IF score in 2015. The total number of articles with the drug discovery theme was only $1 / 5$ of those with the pathophysiological research theme. However, as with pathophysiological research articles, the number and total IF increased in recent years. Compared with the above patterns, it seems that the number and total IF of articles with cell therapy research theme grew steadily from 2006 to 2013, but reached a plateau in 2014-15.

Taking into consideration the above findings, we decided to focus on articles on pathophysiological research and drug discovery based on the growth trend in their numbers in recent years. Because the number of articles on discovery of new drugs was small and the theme of most such articles overlapped with pathophysiological research, we decided to combine both sets of articles into one group for better analysis. For convenience, we divided the pool of articles to be analyzed into nearly three equal parts in IF order. Its boundary between the top $1 / 3$ and the middle $1 / 3$ was $\mathrm{IF}=6$. The top $1 / 3$ could contain articles with more expected number of citations, in other words, larger influence to produce research trends than the middle $1 / 3$ and the bottom $1 / 3$. In this way, we thought that selecting relatively large influential articles is consistent with our purpose of investigating the research trend with saving labor. So, we decided to analyze only the top $1 / 3$ articles with IF of $\geq 6$ hereafter. As a result, our pool of articles to be analyzed was narrowed down to $39 \%$ actually, yet it covered $\sim 73 \%$ of the total IF score of a whole. 
Table 1. Number of Research Articles with Impact Factor of $\geq 6$ by Development Stage

\begin{tabular}{lrrcc}
\hline & Total & Basic & Applied & Translational \\
\hline Cardiovascular diseases & 40 & 34 & 6 & 0 \\
Neurological diseases & 104 & 98 & 6 & 0 \\
Musculoskeletal diseases & 7 & 6 & 1 & 0 \\
Digestive tract diseases & 14 & 12 & 2 & 0 \\
Immunological/hematological & 41 & 41 & 0 & 0 \\
$\quad$ diseases & 2 & 1 & 1 & 0 \\
Urological diseases & 4 & 4 & 0 & 0 \\
Respiratory diseases & 5 & 5 & 0 & 0 \\
Ophthalmological diseases & 43 & 41 & 2 & 0 \\
Miscellaneous & 251 & 233 & 18 & 0 \\
Sum & & &
\end{tabular}

aThe sum does not always match up to "Sum" because it counts articles spanning multiple disease areas in duplicate.

Analysis based on the development stage

Among articles with IF of $\geq 6$ classified with the pathophysiological research and/or drug discovery theme, those on neurological diseases were the most frequent, followed by those on cardiovascular, immunological/ hematological, and digestive tract diseases. Articles on the urinary tract, respiratory, and ophthalmological diseases were the least. These articles were further classified into three categories; basic, applied, and translational research, according to the development stage. Surprisingly, no articles were published on translational research (preclinical and clinical), whereas several dealt with research that focused mainly on disorders of the cardiovascular and nervous systems (Table 1). In the cardiovascular area, the six applied research articles dealt with the topics of long QT syndrome, ${ }^{4-6}$ cardiotoxicity, ${ }^{7}$ drug-induced arrhythmias, ${ }^{8}$ and catecholaminergic polymorphic ventricular tachycardia. ${ }^{9}$ In the neurological disease field, six applied research articles dealt with Parkinson's disease, ${ }^{10,11}$ Down's syndrome, ${ }^{12}$ familial dysautonomia, ${ }^{13}$ amyotrophic lateral sclerosis (ALS), ${ }^{14}$ and paclitaxelinduced sensory peripheral neuropathy. ${ }^{15}$

\section{Analysis based on the publication year}

Figure 2 shows the number of articles with IF of $\geq 6$ stratified according to body systems and publication year. The number of articles on cardiovascular, neurological, and immunological/hematological diseases increased steadily since 2010. Research on liver, which is important as it evaluates toxicity, started to increase since 2014.

Interestingly, the pattern of total IF according to the publication year was similar to that of number of publications (not shown).

Analysis based on country of corresponding author Figure 3 shows the results of analysis of the number of publications with IF of $\geq 6$ by country. The highest number $(n=161)$ of articles were published by scientists from the United States. The number of publications on iPSCs from the United States far exceeded those from Japan, which came second, followed by Germany and the United Kingdom. Other countries included Israel, China, South Korea, and France.

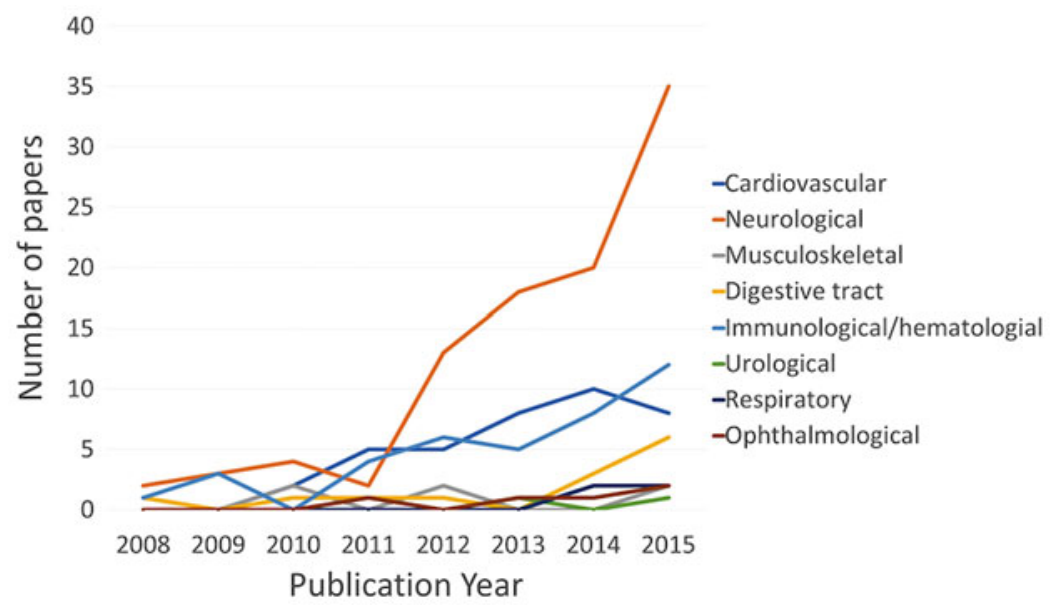

FIG. 2. Number of iPSC-based research articles on PubMed database stratified by body organs and publication year. Data represent all research articles on pathophysiological causes of diseases and discovery of new drugs, published in peer-reviewed journals with IF of $\geq 6$. In this graph, articles published in 2016 were not included since they could not include all articles published in the entire year. 


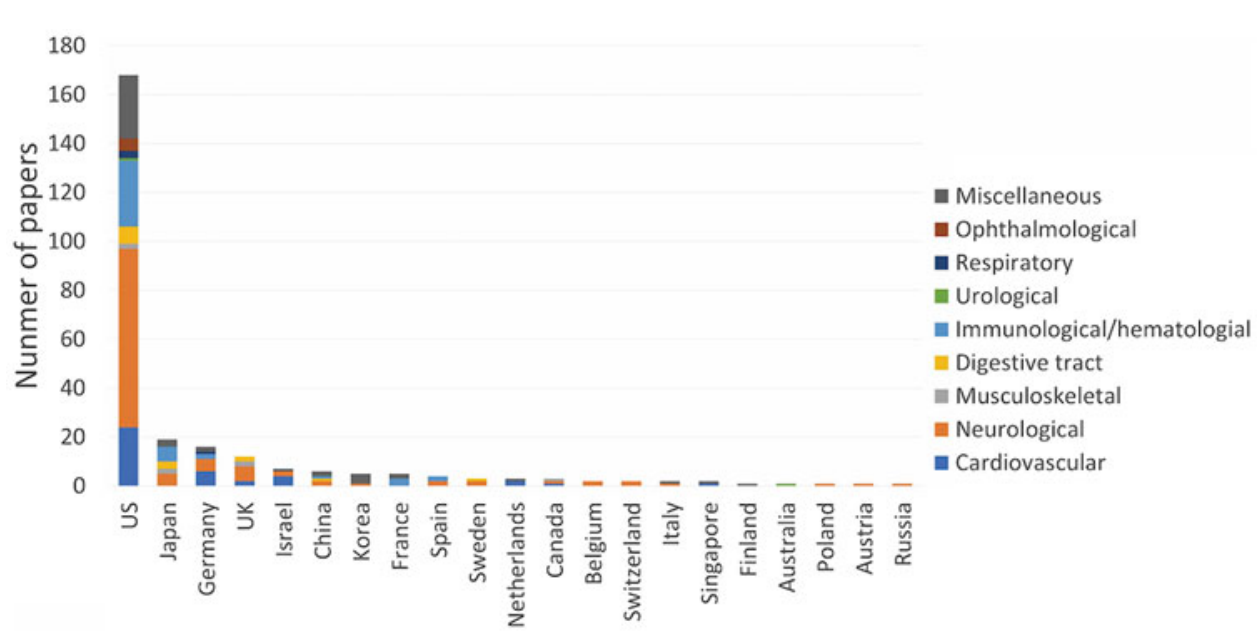

FIG. 3. Number of iPSC-based research articles on PubMed database stratified by country and targeted disease areas. All pathophysiological research/drug discovery articles published in peer-reviewed journals with IF of $\geq 6$ were allocated by individual nations according to the affiliation of the corresponding author. Their targeted disease areas are shown as stacked column.

Figure 4 shows the share of total IF score in pathophysiological research/drug discovery articles with IF of $\geq 6$ according to diseases of body systems. Analysis of the total IF scores, that is, the mass (quality $\times$ quantity) of research, showed the following order: neurological diseases $>$ cardiovascular diseases $>$ immunological/ hematological diseases $>$ digestive tract diseases (Fig. 4). The mass of research on neurological diseases was twice as large (top rank) as that on cardiovascular diseases (second rank) and about 10 times that of the digestive tract diseases (fourth rank), a trend almost similar to that of numbers of publications.

The pattern of ranking of nations according to total IF score (Fig. 5) was almost same as the number of publications shown in Figure 3. The total IF score for publications from the United States was 2825, about 13 times

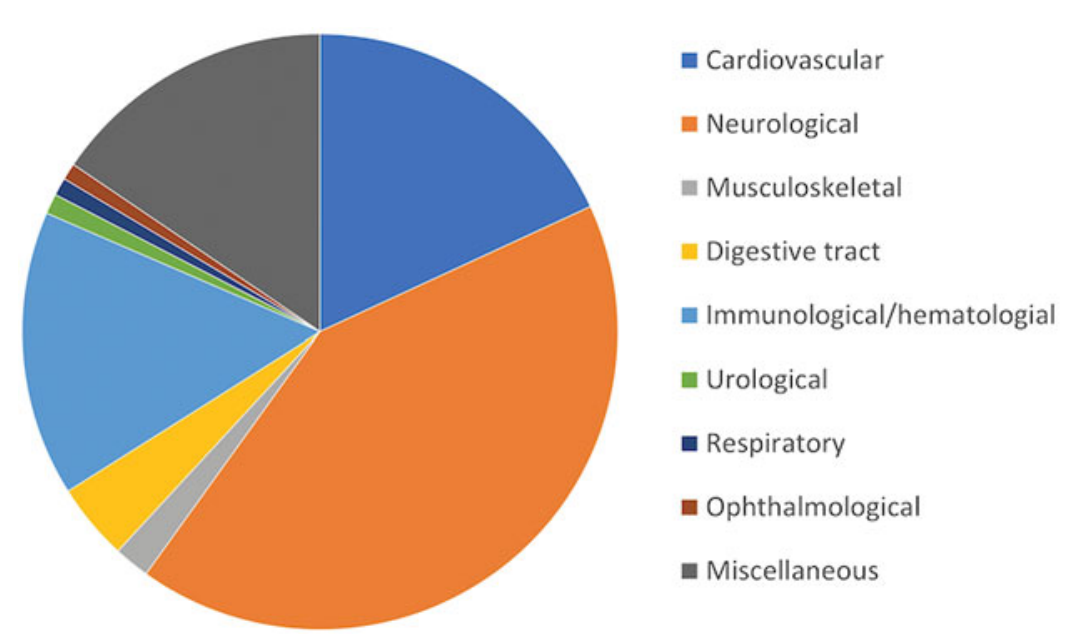

FIG. 4. Global share of total IF score of original research articles in PubMed database by targeted disease areas. Data represent all pathophysiological research/drug discovery articles published in journals with IF of $\geq 6$. The total IF scores of individual targeted disease areas were summed and shown as pie chart. 


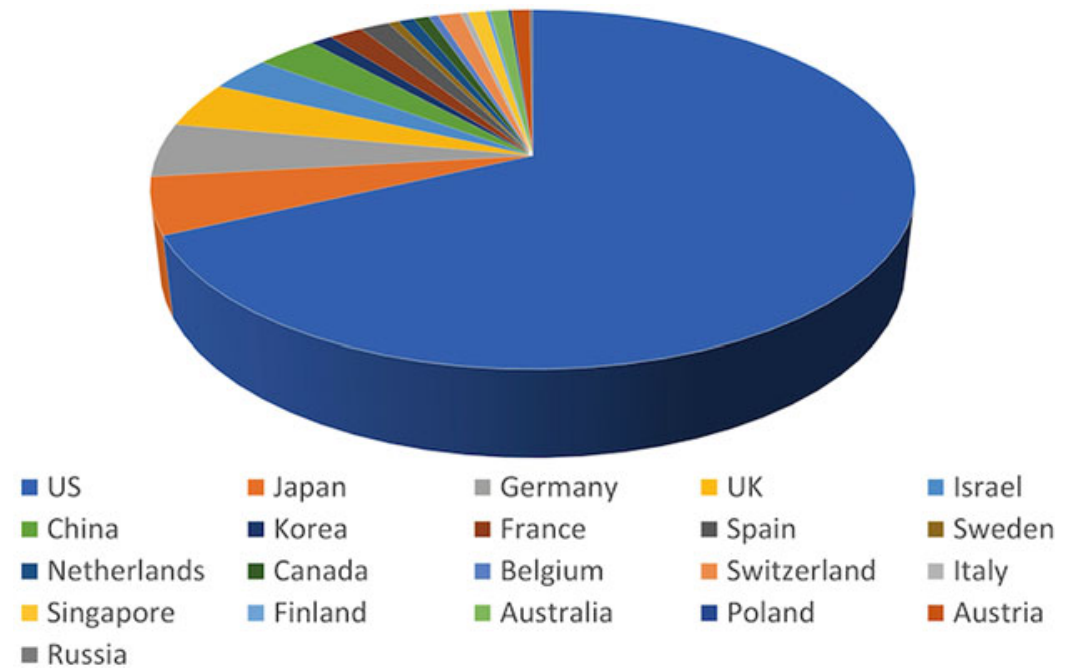

FIG. 5. Global share of total IF score of original research articles in PubMed database by country. Data represent all pathophysiological research/drug discovery articles published in journals with IF of $\geq 6$. The total IF scores of individual nations were summed and shown as a 3D pie chart. 3D, three-dimensional.

that of Japan, which ranked the second. South Korea, which ranked seventh by the number of research articles, moved down to the 10th place on total IF score. Further analysis of the research area according to country (Fig. 6) showed that research work involving iPSCs in the United States focused on extremely wide variety of neurological diseases (e.g., Parkinson's disease, ${ }^{10,11,16-20}$ schizophrenia, ${ }^{21-25}$ Alzheimer's disease, ${ }^{26-30}$ and Huntington's disease), ${ }^{31-35}$ immunological/hematological diseases (e.g., thalassemias, ${ }^{36-39}$ sickle cell anemia, ${ }^{39-41}$
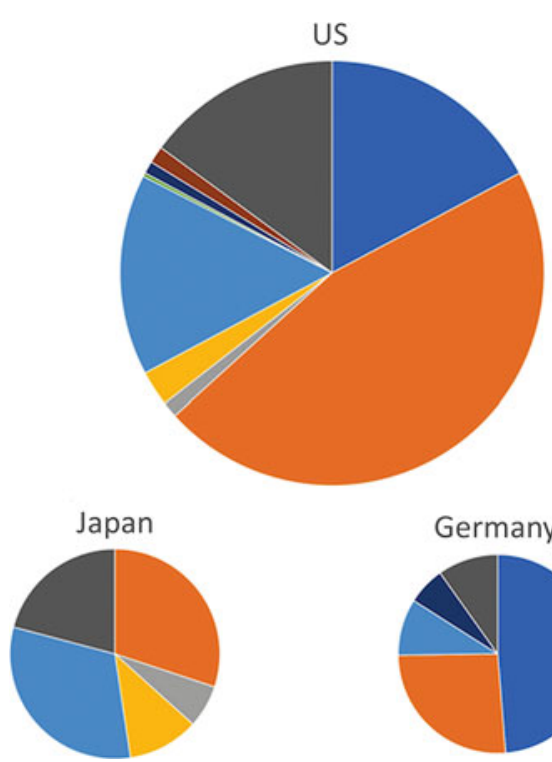

\section{- Cardiovascular \\ neurological \\ Musculoskeletal \\ - Digestive tract \\ n Immunological/hematologial \\ - Urological \\ - Respiratory \\ - Ophthalmological \\ n Miscellaneous}

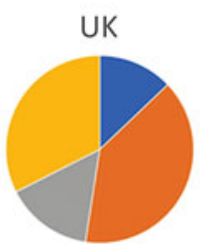

FIG. 6. Country share of total IF score by targeted disease areas of original research articles in PubMed database published by scientists from the United States, Japan, Germany, and United Kingdom. The total IF scores of individual targeted disease areas were summed by country and presented in a pie chart. The pie size represents the rough magnitude of total IF score for each nation. 
and Fanconi anemia), ${ }^{42,43}$ cardiovascular diseases (e.g., arrhythmias, ${ }^{4,5,44-46}$ cardiomyopathy, ${ }^{5,46-51}$ and drug discovery), ${ }^{7,8,52}$ and digestive tract diseases (e.g., $\alpha 1-$ antitrypsin deficiency ${ }^{53-55}$ and drug discovery). ${ }^{56-58}$ Furthermore, the United States made significant pioneering contribution to iPSC-based research on diabetes mellitus ${ }^{59-61}$ and progeria, ${ }^{40,62-64}$ which were classified here as miscellaneous. Work from the United Kingdom also focused on neurological diseases (e.g., ALS), ${ }^{65-67}$ while work from Germany focused on cardiovascular (e.g., arrhythmias) $)^{9,68-70}$ and neurological diseases. ${ }^{71-75}$ Publications by Japanese scientists centered mostly on immunological/hematological ${ }^{76-81}$ and neurological diseases, ${ }^{14,82-85}$ that is, resembling the pattern of the United States. Interestingly, Japanese scientists published two articles on iPSCs in musculoskeletal diseases, ${ }^{86,87}$ which were similar to the numbers of respective articles published by the American and British scientists. It is noteworthy that Japan has so far not published any article on iPSCs in cardiovascular diseases. It should be noted, however, that these are results using only original research articles in relatively large influential journals, that is, those with IF $\geq 6$.

\section{Discussion}

In their study on research trends on human embryonic stem cells (ESCs) and iPSCs, Kobold et al. ${ }^{88}$ evaluated the relevance between studies on both cells in scientific articles published up to 2013. They compared the quality of ESC/iPSC research by calculating the average number of citations per article using Scopus instead of IF. In the present study, we classified articles on iPSCs per main theme without limitation to human iPSCs, evaluated the quality $x$ quantity mass of each theme using total IF score, in addition to the number of articles, and further investigated worldwide research trends, focusing on research on the pathophysiological mechanisms of various diseases and on drug discovery.

We selected articles from the PubMed database published from 2006, when Takahashi and Yamanaka ${ }^{1}$ reported the first iPSC preparation, to the end of August 2016 and analyzed the data.

The results showed that the number and total IF score of articles on iPSC-related reprogramming and differentiation research increased steadily from 2006 to 2013, reached maximum levels in 2013 , and then started to decrease gradually between 2014 and 2015 . On the other hand, the number of pathophysiology research and drug discovery articles increased continuously and forereached those of reprogramming and differentiation research in 2015. This tendency was remarkably evident on the total IF plot (Fig. 1B).

How can we interpret the above data? It is likely that most of the elemental technologies necessary for the preparation of iPSCs and differentiation of iPSCs into the target cells/tissues were investigated and defined in the early days of iPSC research and that research centered on practical and clinical issues, such as pathophysiological mechanisms of diseases and discovery of new drugs (which was developed applying the above technologies), is now entering the maturation stage. In this regard, we performed meta-analysis of the articles dealing with pathophysiological causes of diseases/drug discovery with IF of $\geq 6$. It allowed narrowing the number of articles down to the top 39\% with IF, which still covered $\sim 73 \%$ of the total IF score of a whole.

What about publications on the development stage? Although the number of articles on pathophysiological studies of diseases/drug discovery rose obviously in recent years, the majority of such reports are still at the basic research level, for example, exploring the relationship between gene mutation and disease status, and only a few studies reported concrete results, e.g., practical drug screening and/or discovering new candidate compounds. ${ }^{8,11,13,58,89}$ Among these, we found six applied research articles in the field of cardiovascular and neurological diseases, where the needs of pharmaceutical companies had been high, although it was difficult to obtain human samples.

One article on sensory peripheral neuropathy describing the side effects of paclitaxel ${ }^{15}$ is probably the most practical and clinically oriented report so far, although the study was not a new drug development study, but rather a postmarketing study. This is in contrast to the lack of publications dealing with applications in the field of immunology/hematology. It is possible that this paucity of research is related to the strategy to develop cell therapy using genome editing, instead of conventional drug screening.

Careful analysis of the published articles based on the year of publication identified sufficient number of articles on cardiovascular diseases, neurological diseases, and immunological/hematological diseases published on regular basis dating back before 2010 . Interestingly, the number of articles on neurological diseases increased steeply since 2012; we think that it was, in part, related to the progress and availability of genome editing technology, such as ZFN, TALEN, and CRISPR/Cas 9. On the other hand, the number 
of published articles on the digestive system has also increased in recent years. The main reason suggested is that pharmaceutical companies needed the establishment of cell models reproducing human liver for drug discovery. Analysis of the countries at which iPSC-based research studies were conducted showed that 161 articles with IF of $\geq 6$ (representing $63 \%$ of the selected publications on the pathophysiology of diseases) were published by US scientists. The second group consisted of Japan, Germany, and the United Kingdom, but the combined number of publications with IF of $\geq 6$ from these three countries was less than half of those from the United States.

A close look at the research area by country showed that research from the United States accounts for the majority of publications on cardiovascular, neurological, immunological/hematological, respiratory, and ophthalmological diseases, although there were only a few on musculoskeletal, gastrointestinal, and urological diseases (Fig. 7). In addition, although they were classified under miscellaneous, the United States also majored in iPSC-based publications on diabetes and progeria. Japan, which held the second rank in the number of articles with IF of $\geq 6$, published relatively large number of research articles on immunological/ hematological and neurological diseases. While the number of articles on immunological/hematological diseases showed a presence, those on neurological dis- eases were unremarkable despite ranking third in the number of publications, compared with the United States. Interestingly, about $25 \%$ of the total numbers of articles published on musculoskeletal and digestive diseases were authored by Japanese researchers. However, Japan has so far not published studies on the cardiovascular system with IF of $\geq 6$ in peer-reviewed journals.

Germany, which ranked third in the number of articles with IF of $\geq 6$ on iPSC-based research, ranked second in publications on cardiovascular and respiratory systems, while the United Kingdom, which ranked fourth in the number of articles with IF of $\geq 6$, had the second highest number of publications in the musculoskeletal and digestive fields. Israel, which ranked fifth in the number of published articles, mainly published work on cardiovascular diseases. Furthermore, although Japan ranked second by both the number of articles and total IF score, it is necessary to keep in mind that Japan's position slips to third place when the publications of Germany, France, Spain, and other EU countries are grouped together.

Research projects on the pathophysiology of diseases/drug discovery are closely related to major patient-derived iPSC bank projects in major countries around the world. For example, in the United States, the National Institutes of Health (NIH)-based National Institute of Mental Health (NIMH) Repository and

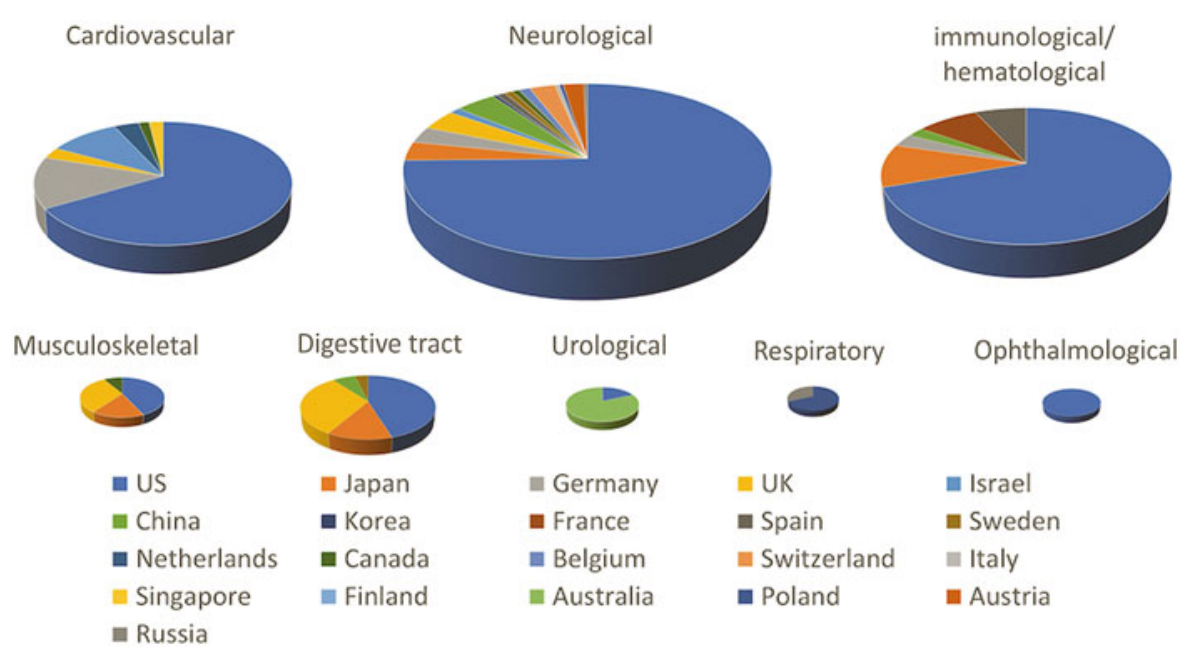

FIG. 7. Share of individual targeted disease areas of total IF score of original research articles in PubMed database by country. The total IF scores of individual nations were summed up by targeted disease areas and presented in a 3D pie chart. The 3D pie size represents the rough magnitude of total IF score for each targeted disease area. 
Genomics Resource (NRGR) and the state-sponsored stem cell research institutes, such as California Institute for Regenerative Medicine (CIRM) and The New York Stem Cell Foundation (NYSCF), produce and deposit patient-derived iPSC lines that focus on various diseases and use them to encourage various research activities on the pathophysiology of diseases and drug discoveries. Looking to Europe, industry-academiclinked patient-derived iPSC bank projects such as the Human Induced Pluripotent Stem Cell Initiative (HipSci) and the European Bank for induced pluripotent Stem Cells (EBiSC) are currently very active. A typical example of a recent article on pathophysiological research of diseases reported identification of the target causative mutation through genetic analysis and the response of cells as detected by in vitro techniques. ${ }^{34}$ Another study provided more direct evidence for the disappearance of a phenotype following gene correction. ${ }^{35}$ In this regard, most of the targeted diseases have so far been rare inheritable diseases. In most articles on immunological/hematological diseases, the combination of pathophysiological research on the disease and gene editing was frequently used to verify the preliminary findings of a series of gene therapy strategies that restored genes in iPSCs derived from the patients, differentiate them into target cells, and infuse them later into the patients.

As discussed above, we believe that research on the pathophysiological mechanisms of diseases can be fruitful when iPSC technology is combined with cell analysis and genome editing. Meanwhile, a recent project named Next Gen studies funded by the National Heart, Lung, and Blood Institute (NHLBI) has tied up with cohort studies and started research on patient-derived iPSCs, which also covers common diseases. This is also a very interesting new clinical research venture.

\section{Conclusion}

Thus, our analysis revealed that the research trend of iPSCs has shifted from research targeting the iPSC itself as reprogramming and differentiation studies to practical application research utilizing iPSC technology as a tool for pathophysiological mechanism of disease and discovery of new drug. It is expected that further steps will be advanced in the future to realize the treatment of human diseases. We hope that the results of research projects currently being developed and conducted around the world can help as many patients as possible.

\section{Acknowledgment}

This work was supported by Highway Program for Realization of Regenerative Medicine of The Japan Agency for Medical Research and Development (AMED).

\section{Author Disclosure Statement}

No competing financial interests exist.

\section{References}

1. Takahashi K, Yamanaka S. Induction of pluripotent stem cells from mouse embryonic and adult fibroblast cultures by defined factors. Cell. 2006;126:663-676.

2. Takahashi K, Tanabe K, Ohnuki M, et al. Induction of pluripotent stem cells from adult human fibroblasts by defined factors. Cell. 2007;131:861-872.

3. Cao Z, Ou C, Teng H, et al. Scientific publications in critical care medicine journals from East Asia: a 10-year survey of the literature. Pak J Med Sci. 2016;32:279-283.

4. Wang $Y$, Liang $P$, Lan F, et al. Genome editing of isogenic human induced pluripotent stem cells recapitulates long QT phenotype for drug testing. J Am Coll Cardiol. 2014;64:451-459.

5. Liang $P$, Lan F, Lee AS, et al. Drug screening using a library of human induced pluripotent stem cell-derived cardiomyocytes reveals diseasespecific patterns of cardiotoxicity. Circulation. 2013;127:1677-1691.

6. Matsa $E$, Rajamohan D, Dick E, et al. Drug evaluation in cardiomyocytes derived from human induced pluripotent stem cells carrying a long QT syndrome type 2 mutation. Eur Heart J. 2011;32:952-962.

7. Feaster TK, Cadar A, Wang L, et al. Matrigel mattress: a method for the generation of single contracting human-induced pluripotent stem cellderived cardiomyocytes. Circ Res. 2015;117:995-1000.

8. Navarrete EG, Liang P, Lan F, et al. Screening drug-induced arrhythmia [corrected] using human induced pluripotent stem cell-derived cardiomyocytes and low-impedance microelectrode arrays. Circulation. 2013;128:S3-S13.

9. Jung $C B$, Moretti $A$, Mederos $Y$, et al. Dantrolene rescues arrhythmogenic RYR2 defect in a patient-specific stem cell model of catecholaminergic polymorphic ventricular tachycardia. EMBO Mol Med. 2012;4:180-191.

10. Woodard CM, Campos BA, Kuo SH, et al. iPSC-derived dopamine neurons reveal differences between monozygotic twins discordant for Parkinson's disease. Cell Rep. 2014;9:1173-1182.

11. Cooper O, Seo H, Andrabi S, et al. Pharmacological rescue of mitochondrial deficits in iPSC-derived neural cells from patients with familial Parkinson's disease. Sci Transl Med. 2012;4:141ra90.

12. Chen $C$, Jiang $P, X u e ~ H$, et al. Role of astroglia in Down's syndrome revealed by patient-derived human-induced pluripotent stem cells. Nat Commun. 2014;5:4430.

13. Lee $\mathrm{G}$, Ramirez $\mathrm{CN}$, Kim H, et al. Large-scale screening using familial dysautonomia induced pluripotent stem cells identifies compounds that rescue IKBKAP expression. Nat Biotechnol. 2012;30:1244-1248.

14. Egawa N, Kitaoka S, Tsukita K, et al. Drug screening for ALS using patientspecific induced pluripotent stem cells. Sci Transl Med. 2012;4:145ra104.

15. Komatsu $\mathrm{M}$, Wheeler HE, Chung $\mathrm{S}$, et al. Pharmacoethnicity in paclitaxelinduced sensory peripheral neuropathy. Clin Cancer Res. 2015;21:4337-4346.

16. Shaltouki A, Sivapatham R, Pei Y, et al. Mitochondrial alterations by PARKIN in dopaminergic neurons using PARK2 patient-specific and PARK2 knockout isogenic iPSC lines. Stem Cell Reports. 2015;4:847-859.

17. Miller JD, Ganat YM, Kishinevsky S, et al. Human iPSC-based modeling of late-onset disease via progerin-induced aging. Cell Stem Cell. 2013;13:691-705.

18. Ryan SD, Dolatabadi N, Chan SF, et al. Isogenic human iPSC Parkinson's model shows nitrosative stress-induced dysfunction in MEF2-PGC1alpha transcription. Cell. 2013;155:1351-1364.

19. Chung CY, Khurana V, Auluck PK, et al. Identification and rescue of alphasynuclein toxicity in Parkinson patient-derived neurons. Science. 2013;342:983-987.

20. Soldner F, Hockemeyer D, Beard C, et al. Parkinson's disease patientderived induced pluripotent stem cells free of viral reprogramming factors. Cell. 2009;136:964-977. 
21. Hook V, Brennand KJ, Kim Y, et al. Human iPSC neurons display activitydependent neurotransmitter secretion: aberrant catecholamine levels in schizophrenia neurons. Stem Cell Rep. 2014;3:531-538.

22. Wen Z, Nguyen HN, Guo Z, et al. Synaptic dysregulation in a human iPS cell model of mental disorders. Nature. 2014;515:414-418

23. Yoon KJ, Nguyen HN, Ursini G, et al. Modeling a genetic risk for schizophrenia in iPSCs and mice reveals neural stem cell deficits associated with adherens junctions and polarity. Cell Stem Cell. 2014;15:79-91.

24. D'Aiuto L, Prasad KM, Upton $\mathrm{CH}$, et al. Persistent infection by HSV-1 is associated with changes in functional architecture of iPSC-derived neurons and brain activation patterns underlying working memory performance. Schizophr Bull. 2015;41:123-132.

25. Tran NN, Ladran IG, Brennand KJ. Modeling schizophrenia using induced pluripotent stem cell-derived and fibroblast-induced neurons. Schizophr Bull. 2013;39:4-10.

26. Akhtar MW, Sanz-Blasco S, Dolatabadi N, et al. Elevated glucose and oligomeric beta-amyloid disrupt synapses via a common pathway of aberrant protein S-nitrosylation. Nat Commun. 2016;7:10242.

27. Young JE, Boulanger-Weill J, Williams DA, et al. Elucidating molecular phenotypes caused by the SORL1 Alzheimer's disease genetic risk factor using human induced pluripotent stem cells. Cell Stem Cell. 2015;16:373 385.

28. Liu Q, Waltz S, Woodruff G, et al. Effect of potent gamma-secretase modulator in human neurons derived from multiple presenilin 1induced pluripotent stem cell mutant carriers. JAMA Neurol. 2014;71:1481-1489.

29. Duan L, Bhattacharyya BJ, Belmadani A, et al. Stem cell derived basal forebrain cholinergic neurons from Alzheimer's disease patients are more susceptible to cell death. Mol Neurodegener. 2014;9:3

30. Israel MA, Yuan SH, Bardy C, et al. Probing sporadic and familial Alz heimer's disease using induced pluripotent stem cells. Nature. 2012;482:216-220.

31. Ring KL, An MC, Zhang N, et al. Genomic analysis reveals disruption of striatal neuronal development and therapeutic targets in human Huntington's disease neural stem cells. Stem Cell Rep. 2015;5:10231038.

32. Lu XH, Mattis VB, Wang N, et al. Targeting ATM ameliorates mutant Huntingtin toxicity in cell and animal models of Huntington's disease. Sci Transl Med. 2014;6:268ra178.

33. Guo X, Disatnik MH, Monbureau M, et al. Inhibition of mitochondrial fragmentation diminishes Huntington's disease-associated neurodegeneration. J Clin Invest. 2013;123:5371-5388.

34. HD iPSC Consortium. Induced pluripotent stem cells from patients with Huntington's disease show CAG-repeat-expansion-associated phenotypes. Cell Stem Cell. 2012;11:264-278.

35. An MC, Zhang N, Scott G, et al. Genetic correction of Huntington's disease phenotypes in induced pluripotent stem cells. Cell Stem Cell. 2012;11:253-263.

36. Xie F, Ye L, Chang JC, et al. Seamless gene correction of beta-thalassemia mutations in patient-specific iPSCs using CRISPR/Cas9 and piggyBac. Genome Res. 2014;24:1526-1533.

37. Chang CJ, Bouhassira EE. Zinc-finger nuclease-mediated correction of alpha-thalassemia in iPS cells. Blood. 2012;120:3906-3914.

38. Papapetrou EP, Lee G, Malani N, et al. Genomic safe harbors permit high beta-globin transgene expression in thalassemia induced pluripotent stem cells. Nat Biotechnol. 2011;29:73-78.

39. Ye L, Chang JC, Lin C, et al. Induced pluripotent stem cells offer new approach to therapy in thalassemia and sickle cell anemia and option in prenatal diagnosis in genetic diseases. Proc Natl Acad Sci U S A 2009;106:9826-9830.

40. Suzuki K, Yu C, Qu J, et al. Targeted gene correction minimally impacts whole-genome mutational load in human-disease-specific induced pluripotent stem cell clones. Cell Stem Cell. 2014;15:31-36.

41. Zou J, Mali $P$, Huang $X$, et al. Site-specific gene correction of a point mutation in human iPS cells derived from an adult patient with sickle cell disease. Blood. 2011;118:4599-4608.

42. Chlon TM, Ruiz-Torres S, Maag L, et al. Overcoming pluripotent stem cell dependence on the repair of endogenous DNA damage. Stem Cell Rep. 2016;6:44-54

43. Muller LU, Milsom MD, Harris $C E$, et al. Overcoming reprogramming resistance of Fanconi anemia cells. Blood. 2012;119:5449-5457.
44. Spencer $\mathrm{Cl}$, Baba $\mathrm{S}$, Nakamura $\mathrm{K}$, et al. Calcium transients closely reflect prolonged action potentials in iPSC models of inherited cardiac arrhythmia. Stem Cell Rep. 2014;3:269-281.

45. Ma Z, Koo S, Finnegan MA, et al. Three-dimensional filamentous human diseased cardiac tissue model. Biomaterials. 2014;35:1367-1377.

46. Karakikes I, Stillitano F, Nonnenmacher M, et al. Correction of human phospholamban R14del mutation associated with cardiomyopathy using targeted nucleases and combination therapy. Nat Commun. 2015;6:6955.

47. Davis J, Davis LC, Correll RN, et al. A tension-based model distinguishes hypertrophic versus dilated cardiomyopathy. Cell. 2016;165:1147-1159.

48. Hinson JT, Chopra A, Nafissi N, et al.; Heart Disease. Titin mutations in iPS cells define sarcomere insufficiency as a cause of dilated cardiomyopathy. Science. 2015;349:982-986.

49. Wu H, Lee J, Vincent LG, et al. Epigenetic regulation of phosphodiesterases $2 \mathrm{~A}$ and $3 \mathrm{~A}$ underlies compromised beta-adrenergic signaling in an iPSC model of dilated cardiomyopathy. Cell Stem Cell. 2015;17:89-100.

50. Lan $F$, Lee AS, Liang $P$, et al. Abnormal calcium handling properties underlie familial hypertrophic cardiomyopathy pathology in patient-specific induced pluripotent stem cells. Cell Stem Cell. 2013;12:101-113.

51. Sun N, Yazawa M, Liu J, et al. Patient-specific induced pluripotent stem cells as a model for familial dilated cardiomyopathy. Sci Transl Med. 2012:4:130ra47.

52. Riedel M, Jou CJ, Lai S, et al. Functional and pharmacological analysis of cardiomyocytes differentiated from human peripheral blood mononuclear-derived pluripotent stem cells. Stem Cell Rep. 2014;3:131-141.

53. Wilson AA, Ying L, Liesa M, et al. Emergence of a stage-dependent human liver disease signature with directed differentiation of alpha-1 antitrypsindeficient iPS cells. Stem Cell Rep. 2015:4:873-885.

54. Tafaleng EN, Chakraborty S, Han B, et al. Induced pluripotent stem cells model personalized variations in liver disease resulting from alpha1antitrypsin deficiency. Hepatology. 2015;62:147-157.

55. Smith C, Abalde-Atristain L, He C, et al. Efficient and allele-specific genome editing of disease loci in human iPSCs. Mol Ther. 2015;23:570-577.

56. Ma X, Qu X, Zhu W, et al. Deterministically patterned biomimetic human iPSC-derived hepatic model via rapid 3D bioprinting. Proc Natl Acad Sci U S A. 2016;113:2206-2211.

57. Ng S, Schwartz RE, March S, et al. Human iPSC-derived hepatocyte-like cells support Plasmodium liver-stage infection in vitro. Stem Cell Rep. 2015;4:348-359.

58. Shlomai A, Schwartz RE, Ramanan V, et al. Modeling host interactions with hepatitis B virus using primary and induced pluripotent stem cell-derived hepatocellular systems. Proc Natl Acad Sci U S A. 2014;111:12193-12198.

59. Bhatt S, Gupta MK, Khamaisi M, et al. Preserved DNA damage checkpoint pathway protects against complications in long-standing type 1 diabetes. Cell Metab. 2015;22:239-252.

60. lovino S, Burkart AM, Kriauciunas $\mathrm{K}$, et al. Genetic insulin resistance is a potent regulator of gene expression and proliferation in human iPS cells. Diabetes. 2014;63:4130-4142.

61. Hua $\mathrm{H}$, Shang $\mathrm{L}$, Martinez $\mathrm{H}$, et al. iPSC-derived beta cells model diabetes due to glucokinase deficiency. J Clin Invest. 2013;123:3146-3153.

62. Cheung HH, Liu X, Canterel-Thouennon L, et al. Telomerase protects werner syndrome lineage-specific stem cells from premature aging. Stem Cell Rep. 2014;2:534-546.

63. Liu GH, Suzuki K, Qu J, et al. Targeted gene correction of laminopathyassociated LMNA mutations in patient-specific iPSCs. Cell Stem Cell. 2011;8:688-694.

64. Liu GH, Barkho BZ, Ruiz S, et al. Recapitulation of premature ageing with iPSCs from Hutchinson-Gilford progeria syndrome. Nature. 2011:472:221-225.

65. Devlin AC, Burr K, Borooah S, et al. Human iPSC-derived motoneurons harbouring TARDBP or C9ORF72 ALS mutations are dysfunctional despite maintaining viability. Nat Commun. 2015;6:5999.

66. Serio A, Bilican B, Barmada SJ, et al. Astrocyte pathology and the absence of non-cell autonomy in an induced pluripotent stem cell model of TDP43 proteinopathy. Proc Natl Acad Sci U S A. 2013;110:4697-4702.

67. Bilican B, Serio A, Barmada SJ, et al. Mutant induced pluripotent stem cell lines recapitulate aspects of TDP-43 proteinopathies and reveal cellspecific vulnerability. Proc Natl Acad Sci U S A. 2012;109:5803-5808.

68. Malan D, Zhang M, Stallmeyer B, et al. Human iPS cell model of type 3 long QT syndrome recapitulates drug-based phenotype correction. Basic Res Cardiol. 2016;111:14. 
69. Malan D, Friedrichs S, Fleischmann BK, et al. Cardiomyocytes obtained from induced pluripotent stem cells with long-QT syndrome 3 recapitulate typical disease-specific features in vitro. Circ Res. 2011;109:841-847.

70. Moretti A, Bellin M, Welling A, et al. Patient-specific induced pluripotent stem-cell models for long-QT syndrome. N Engl J Med. 2010;363:13971409.

71. Eberhardt E, Havlicek S, Schmidt D, et al. Pattern of functional TTXresistant sodium channels reveals a developmental stage of human iPSCand ESC-derived nociceptors. Stem Cell Rep. 2015;5:305-313.

72. Ehrlich $M$, Hallmann $A L$, Reinhardt $P$, et al. Distinct neurodegenerative changes in an induced pluripotent stem cell model of frontotemporal dementia linked to mutant TAU protein. Stem Cell Rep. 2015;5:83-96.

73. Schondorf DC, Aureli M, McAllister FE, et al. iPSC-derived neurons from GBA1-associated Parkinson's disease patients show autophagic defects and impaired calcium homeostasis. Nat Commun. 2014;5:4028.

74. Mica Y, Lee G, Chambers SM, et al. Modeling neural crest induction, melanocyte specification, and disease-related pigmentation defects in hESCs and patient-specific iPSCs. Cell Rep. 2013;3:1140-1152.

75. Reinhardt $P$, Schmid B, Burbulla LF, et al. Genetic correction of a LRRK2 mutation in human iPSCs links parkinsonian neurodegeneration to ERKdependent changes in gene expression. Cell Stem Cell. 2013;12:354-367.

76. Araki $M$, Yang $Y$, Masubuchi $N$, et al. Activation of the thrombopoietin receptor by mutant calreticulin in CALR-mutant myeloproliferative neoplasms. Blood. 2016;127:1307-1316.

77. Sakurai M, Kunimoto $H$, Watanabe $N$, et al. Impaired hematopoietic differentiation of RUNX1-mutated induced pluripotent stem cells derived from FPD/AML patients. Leukemia. 2014;28:2344-2354.

78. Morishima T, Watanabe K, Niwa A, et al. Genetic correction of HAX1 in induced pluripotent stem cells from a patient with severe congenital neutropenia improves defective granulopoiesis. Haematologica. 2014;99:19-27.

79. Hirata S, Takayama N, Jono-Ohnishi R, et al. Congenital amegakaryocytic thrombocytopenia iPS cells exhibit defective MPL-mediated signaling. J Clin Invest. 2013;123:3802-3814.

80. Tanaka T, Takahashi K, Yamane M, et al. Induced pluripotent stem cells from CINCA syndrome patients as a model for dissecting somatic mosaicism and drug discovery. Blood. 2012;120:1299-1308.

81. Kumano K, Arai S, Hosoi M, et al. Generation of induced pluripotent stem cells from primary chronic myelogenous leukemia patient samples. Blood. 2012;119:6234-6242.
82. Yoshida M, Kitaoka S, Egawa N, et al. Modeling the early phenotype at the neuromuscular junction of spinal muscular atrophy using patient-derived iPSCs. Stem Cell Rep. 2015;4:561-568.

83. Maekawa M, Yamada K, Toyoshima M, et al. Utility of scalp hair follicles as a novel source of biomarker genes for psychiatric illnesses. Biol Psychiatry. 2015;78:116-125

84. Numasawa-Kuroiwa Y, Okada Y, Shibata S, et al. Involvement of ER stress in dysmyelination of Pelizaeus-Merzbacher disease with PLP1 missense mutations shown by iPSC-derived oligodendrocytes. Stem Cell Rep. 2014;2:648-661.

85. Kondo T, Asai M, Tsukita K, et al. Modeling Alzheimer's disease with iPSCs reveals stress phenotypes associated with intracellular Abeta and differential drug responsiveness. Cell Stem Cell. 2013;12:487-496.

86. Li HL, Fujimoto N, Sasakawa N, et al. Precise correction of the dystrophin gene in duchenne muscular dystrophy patient induced pluripotent stem cells by TALEN and CRISPR-Cas9. Stem Cell Rep. 2015;4:143-154.

87. Kazuki $Y$, Hiratsuka M, Takiguchi $M$, et al. Complete genetic correction of ips cells from Duchenne muscular dystrophy. Mol Ther. 2010;18:386-393.

88. Kobold S, Guhr A, Kurtz A, et al. Human embryonic and induced pluripotent stem cell research trends: complementation and diversification of the field. Stem Cell Rep. 2015;4:914-925.

89. Shang $L$, Hua $H$, Foo $K$, et al. Beta-cell dysfunction due to increased ER stress in a stem cell model of Wolfram syndrome. Diabetes. 2014;63:923-933.

Cite this article as: Negoro T, Okura H, Matsuyama A (2017) Induced pluripotent stem cells: global research trends, BioResearch Open Access 6:1, 63-73, DOI: 10.1089/biores.2017.0013.

$\begin{aligned} & \text { Abbreviations Used } \\ \mathrm{ALS} & =\text { amyotrophic lateral sclerosis } \\ \mathrm{ESC} & =\text { embryonic stem cell } \\ \mathrm{IF} & =\text { impact factor } \\ \mathrm{iPSC} & =\text { induced pluripotent stem cell } \\ \mathrm{n} & =\text { number } \\ \mathrm{S} & =\text { score }\end{aligned}$

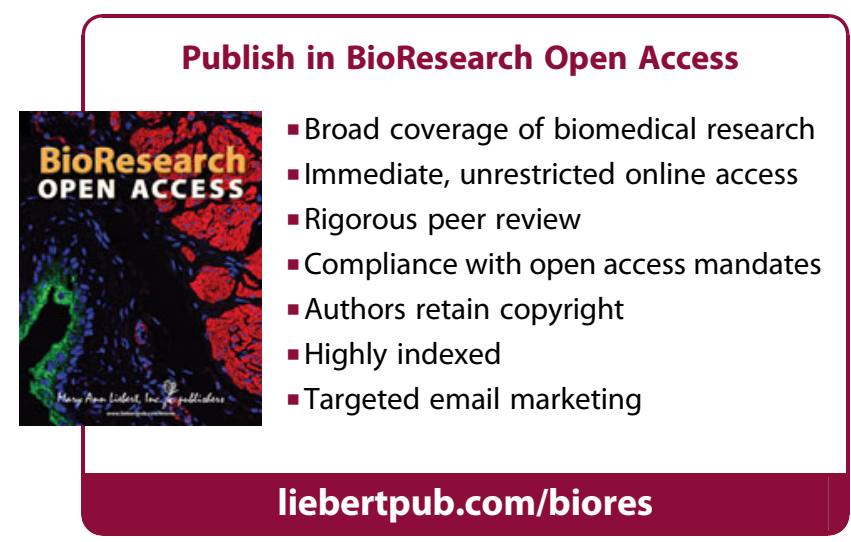

